\title{
KALORIMETRISCHE VERFAHREN BEI HÖHEREN TEMPERATUREN
}

\author{
Franz Eberhard Wittig \\ Physikalisch-Chemisches Institut der Universität, München, Deutschland
}

Bei kalorimetrischen Bestimmungen von Bildungswärmen, Wärmeinhalten und Umwandlungswärmen werden in der Regel diejenigen Verfahren bevorzugt, die eine Messung dieser Grössen bei Zimmertemperatur gestatten, weil diese Methoden sehr genaue Ergebnisse liefern und mit einem geringeren apparativen Aufwand auskommen. In manchen Fällen lassen sich diese bekannten und bewährten Verfahren ${ }^{1-4}$ aber nicht in befriedigender Weise oder überhaupt nicht anwenden, so daß kalorimetrische Methoden bei höheren Temperaturen notwendig werden ${ }^{5}$.

Bildungswärmen werden in der Regel nicht durch direkte Synthese, sondern indirekt aus Verbrennungs- und Lösungswärmen bestimmt. Dabei kann der Fall auftreten, daß die Bildungswärme als sehr kleine Differenz grosser Zahlen und daher auch bei großer Präzision der Messungen nur sehr ungenau bestimmt wird. Trotz der etwas geringeren Genauigkeit der Hochtemperaturkalorimeter (H.T.K.) kann dann ein anderer Reaktionsweg oder die direkte Synthese bei höheren Temperaturen zu besseren Ergebnissen führen. Insbesondere bei intermetallischen Verbindungen lassen sich vielfach bei Zimmertemperaturen keine brauchbaren Reaktionen auffinden, so daß systematische Untersuchungen nur durch Lösungskalorimetrie in flüssigen Metallen ausgeführt werden können. Manche Reaktionen, wie etwa die Mischung flüssiger Metalle, lassen sich überhaupt nur bei höheren Temperaturen verfolgen. Das gilt auch für den Übergang von durch Abschrecken erhaltenen Nichtgleichgewichten in thermodynamisches Gleichgewicht, wobei nicht nur die Wärmetönung, sondern auch die Kinetik von Interesse ist. Wärmeinhalte, spezifische Wärmen und Umwandlungswärmen können nach dem Mischungsverfahren (drop method) oder mit Oelsens Abkühlungskalorimeter prinzipiell nur dann einwandfrei gemessen werden, wenn die Umwandlungen bei der Abkühlung quantitativ ablaufen und die Probe sich nach vollständiger Abkühlung im thermodynamischen Gleichgewicht befindet. Wenn diese Bedingung nicht erfüllt ist, müssen die genannten Größen direkt bei höheren Temperaturen bestimmt werden. Direkte Messungen der wahren spezifischen Wärme bei höheren Temperaturen sind auch dann angebracht, wenn diese Größe sich aus Messungen des Wärmeinhaltes als Differentialquotient nicht mit ausreichender Genauigkeit ableiten läßt.

Mit diesen Beispielen ist der heutige Anwendungsbereich1, 3-5, 8, 9, 89 der H.T.K. etwa umrissen. Die Technik der Kalorimetrie bei höheren Temperaturen bietet aber im Vergleich zu den bei Zimmertemperatur bekannten und üblichen Verfahren einige zusätzliche experimentelle Schwierigkeiten, die im folgenden kurz behandelt werden sollen. Ausserdem werden für alle Anwendungsbereiche die wichtigsten technischen Merkmale bewährter 
Konstruktionen angegeben; eine ausführliche Darstellung aller bekannten Apparaturen soll an anderer Stelle erscheinen ${ }^{6}$. Wegen der einfacheren, aber zumeist auch weniger genauen Verfahren der Differentiellen Thermoanalyse (D.T.A.) sei auf eine Monographie von Smothers und Chiang ${ }^{7}$ (mit 1546 Literaturzitaten bis 1957) verwiesen.

\section{ALLGEMEINER TEIL}

\subsection{Die Kalorimetergleichung}

In allgemeinen ist ein Kalorimeter ein zur Aufnahme eines Reaktionsgefässes bestimmter Hohlkörper, dessen Temperatur $T_{\mathrm{k}}$ entsprechend genau bestimmt werden kann. Im Kalorimeter kann elektrisch oder chemisch eine zeitlich variable oder konstante Wärmeleistung $L(z)$ (in W) entwickelt werden. Der Wasserwert (energy equivalent) ist eine temperaturabhängige Konstante $W(T)$, welche diejenige Wärmemenge $\left(\mathrm{J} /{ }^{\circ} \mathrm{C}\right)$ bezeichnet, die notwendig ist, um bei adiabatischer Arbeitsweise, also ohne Wärmeaustausch mit der Umgebung, die Temperatur $T_{k}$ des Kalorimeters um $1^{\circ} \mathrm{C}$ zu erhöhen. Das Kalorimeter ist von einem Thermostaten der konstant (oder nach einem bestimmten Programm variabel) geregelten Temperatur $T_{t}$ umgeben. Eine weitere temperaturabhängige Apparatekonstante $K(T)$ bezeichnet den sekundlichen Wärmeaustausch des Kalorimeters mit dem Thermostaten $\left(\mathrm{W} /{ }^{\circ} \mathrm{C}\right)$ bei einer Temperaturdifferenz von $T_{\mathrm{k}}-T_{\mathrm{t}}=1$. Die in der Zeit $\mathrm{d} z$ dem Kalorimeter zugeführte Wärmemenge $L(z) \mathrm{d} z$ wird dann gemäss der Kalorimetergleichung erster Näherung

$$
L(z) \mathrm{d} z=W(T) \mathrm{d} T_{\mathrm{k}}+K(T)\left(T_{\mathrm{k}}-T_{\mathrm{t}}\right) \mathrm{d} z
$$

in zwei Anteile aufgespalten, nämlich in eine Erwärmung des Kalorimeters um $\mathrm{d} T_{\mathrm{k}}$ und einen Wärmeaustausch mit dem Thermostaten.

Reaktionswärmen im Kalorimeter werden also aus dem beobachteten Verlauf von $T_{\mathrm{k}}$ und $T_{\mathrm{t}}$, sowie den gesondert bestimmten Apparatekonstanten $W$ und $K$ als Integral über die nach Gl. (I) berechnete Leistung bestimmt.

Der häufigste Fall ist durch einen isothermen Thermostaten mit $T_{t}=$ konst gegeben. Befindet sich dabei das Kalorimeter zur Zeit $z=0$ auf einer von $T_{t}$ verschiedenen Anfangstemperatur $T^{\circ}$ und findet gemäss $L=0$ keine Wärmeentwicklung statt, so folgt mit

$$
W(T) \mathrm{d} T_{\mathrm{k}}=-K(T)\left(T_{\mathrm{k}}-T_{\mathrm{t}}\right) \mathrm{d} z
$$

eine Temperaturänderung des Kalorimeters durch Wärmeaustausch mit der Umgebung. Für die zeitliche Anderung von $T_{\mathrm{k}}$ folgt dann mit

$$
T_{\mathrm{k}}=T_{\mathrm{t}}+\left(T^{\circ}-T_{\mathrm{t}}\right) \exp (-K z / W)
$$

ein exponentieller Ausgleich der Temperaturen, dessen zeitlicher Verlauf durch die Zeitkonstante $Z_{\mathrm{k}}=W / K$ gegeben ist. Bei Zimmertemperatur ist bei nicht zu kleinem Wasserwert $W$ und, in der Regel, kleinem $K$ die Zeitkonstante $Z_{\mathrm{k}}$ sehr gross, so dass die Reihenentwicklung von Gl. (3) mit dem linearen Glied abgebrochen werden kann. Wir erhalten dann

$$
T_{\mathrm{k}}=T_{\mathrm{t}}+\left(T^{\circ}-T_{\mathrm{t}}\right)(1-K z / W)=T^{\circ}-\left(T^{\circ}-T_{\mathrm{t}}\right) K z / W
$$




\section{KALORIMETRISCHE VERFAHREN}

Werden alle $Z_{\mathrm{p}}$ sek äquidistante Ablesungen ausgeführt, so wird $z=n \cdot Z_{\mathrm{p}}(n=0,1,2,3 \ldots)$ und wir erhalten aus Gl. (4)

$$
T_{\mathrm{k}}=T^{\circ}-\left(T^{\circ}-T_{\mathrm{t}}\right) n\left(Z_{\mathrm{p}} K / W\right)=T^{\circ}-\left(T^{\circ}-T_{\mathrm{t}}\right) n k
$$

Die konventionelle thermochemische Abkühlungskonstante, $k$, ergibt sich also durch Einbeziehung der Dauer $z_{p}$ einer Ableseperiode in die Zeitkonstante $Z_{\mathrm{k}}$ gemäss

$$
k=K, Z_{\mathrm{p}} / W=Z_{\mathrm{p}} / Z_{\mathrm{k}}
$$

Die Temperaturänderung $\Delta T_{\mathrm{k}}$ in einer Ableseperiode wird als Gang bezeichnet. Bei der üblichen Auswertung wird allerdings die Anfangstemperatur $T^{\circ}$ nicht ganz konsequent durch die mittlere Temperatur in Vor- oder Nachperiode $T_{\mathrm{v}}$ oder $T_{\mathrm{n}}$ ersetzt, und anstelle der Thermostatentemperatur $T_{t}$ eine rechnerische Konvergenztemperatur $T_{0}$ eingeführt, in der auch der Einfluss von sekundären Effekten (z.B. Rühr- oder Verdampfungswärmen) enthalten ist. Wir erhalten dann für die Gänge in Vorund Nachperiode

$$
G_{\mathrm{v}}=-k\left(T_{\mathrm{v}}-T_{\mathrm{o}}\right) \text { und } G_{\mathrm{n}}=-k\left(T_{\mathrm{n}}-T_{\mathrm{o}}\right)
$$

Bei höheren Temperaturen nimmt nun $K(T)$ schnell zu; als grobe Faustregel lässt sich eine Verdopplung für je $200^{\circ} \mathrm{C}$ annehmen. Die Forderung definierter Temperaturverteilung führt ausserdem zu kleinen Abmessungen und, in Verbindung mit den an sich schon geringen Wärmeinhalten metallischer Kalorimeter, zu kleinen Wasserwerten $W(T)$. Die Zeitkonstante $Z_{\mathrm{k}}$ wird also wesentlich kleiner, so dass die lineare Näherung Gl. (4) nicht mehr erlaubt ist. Wir erhalten dann aus Gl. (3)

$$
\ln \left(T_{\mathrm{k}}-T_{\mathrm{t}}\right)=\ln \left(T^{\circ}-T_{\mathrm{t}}\right)-n k
$$

Bei logarithmischer Darstellung resultiert daher ein linearer Verlauf, dessen Achsenabschnitt für $n=0$ durch $\ln \left(T^{\circ}-T_{t}\right)$ und dessen Neigung durch $k$ gegeben ist. Im Gegensatz zu Gl. (7) ist der logarithmische Gang also nicht mehr durch $T^{\circ}$, sondern lediglich durch $k$ bedingt.

\subsection{Bestimmung der Konstanten $k, K$, und $W$}

\subsection{Bestimmung von $k$}

Bei grossen Zeitkonstanten lässt sich $k$ nach Gl. (7) aus dem Gang in der Vor- oder Nachperiode bestimmen. Da aber die rechnerische Konvergenztemperatur $T_{\mathrm{o}}$ nicht bekannt ist, wird sie eliminiert, so dass wir

$$
k=\left(G_{\mathrm{v}}-G_{\mathrm{n}}\right) /\left(T_{\mathrm{n}}-T_{\mathrm{v}}\right)
$$

erhalten. Für sehr genaue Messungen empfiehlt sich die einfache Berechnung von $G_{\mathrm{v}}$ und $G_{\mathrm{n}}$ durch lineare Ausgleichsrechnung äquidistanter Werte.

Bei höheren Temperaturen und kleineren Zeitkonstanten muss dagegen die exponentielle Krümmung nach Gl. (3) und (8) berücksichtigt werden. $T_{t}$ lässt sich dann aber nicht mehr in einfacher Weise eliminieren. Es ist 
aber ohnehin erforderlich, die Messung erst einzuleiten, wenn sich nach Einbau einer Probe im Kalorimeter wieder ein thermisches Gleichgewicht eingestellt hat. Bei den in Frage kommenden kleineren Zeitkonstanten befindet sich dass Kalorimeter dann aber auch im Gleichgewicht mit dem Thermostaten, so dass $T_{t}$ sich aus dem Mittelwert einer Anzahl von Ablesungen in der Vorperiode ergibt und in Gl. (8) eingesetzt werden kann; $k$ folgt dann aus dem logarithmischen Gang nach Gl. (8).

Bei kleinen Zeitkonstanten ist die Genauigkeit von $k$ vor allem durch die Schwankungen des Nullpunktes $\boldsymbol{T}_{\mathbf{t}}$ begrenzt, woraus sich sofort die Notwendigkeit einer sehr konstanten Regelung von $T_{t}$ ergibt. Dabei entsteht aber noch eine weitere für H.T.K. kennzeichnende Schwierigkeit. Wegen der zumeist kleinen Abmessungen des Kalorimeters ist der Wasserwert $W_{\mathrm{r}}$ des Reaktionsraumes nicht mehr klein im Vergleich mit $W(T)$, so dass die zweite Zeitkonstante $W_{\mathrm{r}} / K_{\mathrm{r}}$ berücksichtigt werden muss und die Gleichung erster Näherung (1) das zeitliche Verhalten nicht mehr hinreichend genau beschreibt. In der Nachperiode macht dieser Umstand sich durch anfängliche Abweichungen vom logarithmischen Verlauf nach Gl. (8) bemerkbar, so dass $k$ erst aus späteren, und damit auch kleineren und stärker durch die Nullpunktsschwankungen beeinträchtigten Ablesungen der Temperaturdifferenz $\left(T_{k}-T_{t}\right)$ bestimmt werden muss.

\subsection{Bestimmung von $K$}

Die Konstante $K$ wird nach dem Verfahren der Gleichgewichtsleistung bestimmt ${ }^{31}$. Dazu wird das Kalorimeter bei konstantem $T_{t}$ auf eine bestimmte höhere Temperatur $T_{\mathrm{k}}$ aufgeheizt, und eine Leistung $L$ mit nur noch möglichst geringen Änderungen von $T_{\mathrm{k}}$ elektrisch erzeugt. Nach Einstellung des thermischen Gleichgewichts wird $T_{\mathrm{k}}$ als Mittelwert einiger äquidistanter Ablesungen bestimmt. Da im Gleichgewicht $\mathrm{d} \mathcal{T}_{\mathrm{k}}=\mathrm{O}$, folgt aus Gl. (1)

$$
K(T)=L /\left(T_{\mathrm{k}}-T_{\mathrm{t}}\right)
$$

Zweckmässigerweise wird diese Bestimmung bei verschiedenen Werten von $T_{\mathrm{k}}$ und $L$ ausgeführt und $K(T)$ durch lineare Ausgleichsrechnung ermittelt. In dieser Weise lässt sich gegebenenfalls auch feststellen, bis zu welchen maximalen Werten von $\left(T_{\mathrm{k}}-T_{\mathrm{t}}\right)$ die Temperaturabhängigkeit von $K(T)$ noch vernachlässigt werden kann ${ }^{5}$.

Bei bekanntem $k$ und $W$ lässt sich zur Kontrolle $K$ auch gemäss Gl. (6) berechnen.

\subsection{Bestimmung von $W(T)$}

Wenn $K$ und $k$ nach den vorstehenden Methoden bestimmt wurden, lässt sich $W(T)$ nach GI. (6) zu $W(T)=K Z_{\mathrm{p}} / k$ berechnen. Im allgemeinen muss $W(T)$ aber wesentlich genauer bestimmt werden, als es nach dieser Formel möglich ist. Bei adiabatischen Kalorimetern ergibt sich $W(\mathcal{T})$ definitionsgemäss als Verhältnis der auf elektrischem Wege zugeführten und genau gemessenen Wärmemenge und der adiabatisch bestimmten Temperaturerhöhung $T_{e}-T_{a}$. Vielfach besteht aber nicht die Möglichkeit einer hinreichend genauen adiabatischen Regelung. In 


\section{KALORIMETRISGHE VERFAHREN}

diesem Fall muss die mit dem Thermostaten der konstanten Temperatur $T_{\mathrm{t}}$ ausgetauschte Wärmemenge $Q_{\mathrm{a}}$ berücksichtigt werden. Diese ergibt sich aus den $\mathcal{N}$ Ablesungen in der Hauptperiode durch Integration des Wärmeaustauschgliedes der Gl. (1) $K\left(T_{\mathrm{k}}-T_{\mathrm{t}}\right) \mathrm{d} z$ nach der Trapezregel zwischen der letzten Ablesung $T_{\mathrm{a}}$ der Vorperiode über die $\mathcal{N}$ Ablesungen $T_{\mathrm{n}}$ der Hauptperiode bis zur ersten Ablesung der Nachperiode $T_{\mathrm{e}} \mathrm{zu}$

$$
Q_{\mathrm{a}}=K\left[\left(T_{\mathrm{a}} / 2+\Sigma T_{\mathrm{n}}+T_{\mathrm{e}} / 2\right)-(\mathcal{N}+1) T_{\mathrm{t}}\right] Z_{\mathrm{p}}
$$

In der Regel wird aber nicht $K$ nach Abschnitt 1.22, sondern $k=K Z_{\mathfrak{p}} / W$ aus den Gängen nach Abschnitt 1.22 bestimmt. Durch Division von Gl. (12) mit $W(T)$ führen wir statt $K Z_{\mathrm{p}}$ die Konstante $k$ ein, was eine Umrechnung von $Q_{a}$ auf eine Korrektur der Temperaturänderung $\Delta T$ bedeutet. So erhalten wir

$$
Q_{\mathrm{a}} / W=\Delta T=k\left[T_{\mathrm{a}} / 2+\Sigma T_{\mathrm{n}}+T_{\mathrm{e}} / 2-(\mathcal{N}+1) T_{\mathrm{t}}\right]
$$

Ausserdem muss $T_{t}$ durch die rechnerische Konvergenztemperatur $T_{0}$ ersetzt, und diese nach Gl. (7) eliminiert werden. Mit

$$
k T_{\mathrm{o}}=G_{\mathrm{v}}+k T_{\mathrm{v}}=G_{\mathrm{n}}+k T_{\mathrm{n}}
$$

erhalten wir dann aus Gl. (12) die beiden Formen der Regnault-PfaundlerKorrektur

$$
\begin{aligned}
\Delta T & =k\left[T_{\mathrm{a}} / 2+\Sigma T_{\mathrm{n}}+T_{\mathrm{e}} / 2-(\mathcal{N}+1) T_{\mathrm{v}}\right]-(\mathcal{N}+1) G_{\mathrm{v}} \\
& =k\left[T_{\mathrm{a}} / 2+\Sigma T_{\mathrm{n}}+T_{\mathrm{e}} / 2-(\mathcal{N}+1) T_{\mathrm{n}}\right]-(\mathcal{N}+1) G_{\mathrm{n}}
\end{aligned}
$$

Der zweite Ausdruck bietet den Vorteil, dass $k$ mit einer kleineren Zahl multipliziert wird. Es empfiehlt sich aber, die Berechnung ausserdem nach der ersten Form auszuführen, was bei einer geringen Vermehrung des Rechenaufwandes eine Kontrolle der Rechnung erlaubt.

Bei höheren Temperaturen wird bei hinreichend kleinen Zeitkonstanten $T_{t}$ in der Regel aus dem Mittelwert der konstanten Ablesungen in der Vorperiode bestimmt. Man kann nun wahlweise nach Abschnitt 1.21 die Konstante $k$ aus dem logarithmischen Gang $n k$ in der Nachperiode nach Gl. (8) ableiten und aus Gl. (12) die Korrektur der Temperaturerhöhung berechnen, oder $K$ nach Abschnitt 1.22 bestimmen und dann nach Gl. (11) direkt die ausgetauschte Wärmemenge $Q_{\mathrm{a}}$ ableiten und als Korrektur an der gemessenen Wärmetönung anbringen.

Macht sich in der Nachperiode die zweite Zeitkonstante des Reaktionsgefässes $Z_{\mathrm{kr}}=W_{\mathbf{r}} / K_{\mathrm{r}}$ derart bemerkbar, dass der lineare Verlauf nach Gl. (8) sich nicht einstellt, so empfiehlt sich eine andere Auswertung. Dazu wird die Temperatur des Kalorimeters $T_{\mathrm{k}}$ in äquidistanten Ablesungen bis zur Einstellung des thermischen Gleichgewichts verfolgt und die Reaktionswärme ausschliesslich nach Gl. (11) als mit dem Thermostaten ausgetauschte Warmemenge $Q_{a}$ bestimmt, wozu natürlich eine vorherige Messung von $K$ nach Abschnitt 1.22 erforderlich ist. Dieses Verfahren ist vor allem bei kleinen Zeitkonstanten praktikabel, weil die Messgenauigkeit durch die Schwankungen des Nullpunktes beeinträchtigt wird, und 
daher die Streuungen umso kleiner werden, je schneller der Versuch abläuft. In dieser Weise lassen sich natürlich nur Wärmetönungen, aber keine Wasserwerte bestimmen. Der Wasserwert kann aber mit Vorteil als kleine Korrektur verwendet werden, wenn sich das Kalorimeter nur langsam mit dem Thermostaten ins Gleichgewicht setzt. Es genügt dann, das Produkt dieser kleinen Temperaturdifferenz mit dem Wasserwert als Korrektur an $Q_{\mathrm{a}}$ anzubringen, wozu auch ein näherungsweise gemäss $W=K Z_{\mathrm{p}} / k$ abgeleiteter Wasserwert ausreicht.

Normalerweise wird aber die nach Gl. (12) oder (13) bestimmte Korrektur $\Delta T$ an der Differenz der ersten und letzten Ablesung $T_{\mathrm{e}}-T_{\mathrm{a}}$ angebracht, so dass sich nach Division der elektrisch gemessenen und dem Kalorimeter zugeführten Wärmemenge mit der korrigierten Temperaturerhöhung der Wasserwert ergibt.

Ebenso erhalten wir die unbekannten Reaktionswärmen durch Multiplikation des zuvor bestimmten Wasserwertes mit der korrigierten Temperaturänderung. Bei kleinen Kalorimetern, wie sie vielfach bei höheren Temperaturen verwendet werden, muss natürlich der erhebliche Einfluss des Wärmeinhaltes der im Kalorimeter befindlichen Probe auf den Wasserwert berücksichtigt und bestimmt werden, so dass bei Beschikung mit Proben anderen Wärmeinhaltes eine neue Bestimmung von $W$ erforderlich ist.

\subsection{Die prinzipiellen Möglichkeiten kalorimetrischer Verfahren}

Die vorstehenden Ausführungen bezogen sich überwiegend auf den häufigsten Fall der Erwärmungskalorimetrie, bei dem in einem isothermen Thermostaten der zeitliche Verlauf von $\mathcal{T}_{\mathrm{k}}$ verfolgt wird. In älteren Darstellungen findet man für dieses Verfahren gelegentlich die Bezeichnung 'isotherme Kalorimetrie', etwas irreführend, weil $T_{t}$, nicht aber $T_{\mathbf{k}}$ konstant ist; als isotherm werden wir nur Kalorimeter bezeichnen, bei denen $\mathcal{T}_{\mathrm{k}}$ konstant ist.

Bei der Entwicklung von Hochtemperaturkalorimetern nach dem Erwärmungsprinzip war vor allem die noch nicht geklärte Frage von Interesse, bis $\mathrm{zu}$ welchen Temperaturen dieses Verfahren überhaupt angewendet werden kann. Inzwischen konnten wir die Brauchbarkeit dieser Methode für Genauigkeiten von augenblicklich 1-2 Prozent bei $975^{\circ} \mathrm{C}$ nachweisen $\left(K=1.05 \mathrm{~W} /{ }^{\circ} \mathrm{C}, W=315 \mathrm{~J} /{ }^{\circ} \mathrm{C}, Z_{\mathrm{k}}=300 \mathrm{sec}\right)$. Der Anwendungsbereich lässt sich aber voraussichtlich auf noch höhere Temperaturen und kleinere Zeitkonstanten ausdehnen.

Bei höheren Temperaturen nimmt die Genauigkeit der Erwärmungskalorimetrie ab, weil der Anteil des Wärmeaustausches zunimmt und, wegen der Nullpunktsschwankungen und des schnellen Temperaturausgleichs, $k$ nicht mehr so genau bestimmt werden kann. Es wäre zunächst denkbar, durch adiabatisches Arbeiten den Wärmeaustausch zu unterdrücken, was aber zu prinzipiellen Schwierigkeiten führt und einen wesentlich höheren Regelaufwand (P.I.D.-Regler) bedingt als die isotherme Regelung, die sich auch bei sehr hohen Temperaturen mit einfachen P-Reglern beherrschen lasst. Eine andere Úberlegung beruht darauf, dass die Wärmeentwicklung $L \mathrm{~d} z$ nach Gl. (1) in zwei Anteile, nämlich 


\section{KALORIMETRISCHE VERFAHREN}

der Erwärmung des Kalorimeters und des Wärmeaustausches mit dem Thermostaten zerlegt wird, und der zweite Anteil auf Kosten des ersten bei höheren Temperaturen schnell zunimmt. Es liegt daher näher, den grösseren Anteil des Wärmeaustausches nicht durch adiabatisches Arbeiten zu unterdrücken, sondern zur eigentlichen Messgrösse zu machen und isotherm mit Wärmeströmungskalorimetern zu arbeiten. Dabei lässt sich $K$ durch direkte elektrische Eichung nach 1.22 bestimmen, so dass die dann ungenauere Ableitung von $k$ nach 1.21 entfällt. Wir haben ausserdem mit der Möglichkeit zeitlicher Änderungen von $K$ gerechnet und daher ein automatisches Kompensationsverfahren entwickelt. Bei dieser Methode wird bei konstanter Leistung $L$ mit einem Temperaturregler eine konstante Temperaturdifferenz $T_{\mathrm{k}}-\mathcal{T}_{\mathrm{t}}$ bei konstantem $T_{\mathrm{t}}$ eingestellt, so dass Reaktionswärmen als Differenzen der summierten Einschaltzeiten der Leistung $L$ bestimmt werden können. Dieses Verfahren lässt sich mit Genauigkeiten von 1 Prozent bis augenblicklich $1000^{\circ} \mathrm{C}$ anwenden und dürfte auch bei wesentlich höheren Temperaturen ebenso brauchbar sein, weil es keine temperaturabhängigen Voraussetzungen enthält. Isotherme Verfahren bieten den weiteren Vorteil, dass die Temperaturabhängigkeit von $K(T)$ ausgeschaltet wird.

Wir wollen aber zunächst einmal die prinzipiellen Möglichkeiten kalorimetrischer Verfahren betrachten. Die historisch bedingte Benennung, wie etwa Eiskalorimeter oder Verbrennungskalorimeter, bezieht sich nur auf einzelne und nicht vergleichbare Merkmale und lässt daher die Wirkungsweise des kalorimetrischen Verfahrens nur ungenügend erkennen. Es ist besser, von der Kalorimetergleichung (1) auszugehen, in die neben den Konstanten $W$ und $K$ und der Versuchsdauer $z$ die Variablen $L, T_{\mathrm{k}}$ und $T_{\mathrm{t}}$ eingehen. Die verschiedenen Möglichkeiten einer kalorimetrischen Messung ergeben sich dann aus der Auswahl des Mess- und Regelprogramms dieser Variablen.

Gleichung (1) zeigt nun die erwähnte Aufspaltung der Wärmemenge $L \mathrm{~d} z$ in die Erwärmung des Kalorimeters $W \mathrm{~d} \mathcal{T}_{\mathrm{k}}$ und den Wärmeaustausch $K\left(T_{\mathrm{k}}-T_{\mathrm{t}}\right) \mathrm{d} z$. Daraus folgt sofort die Möglichkeit, entweder den ersten Anteil durch isothermes Arbeiten wegen $\mathrm{d} T_{\mathrm{k}}=0$, oder den zweiten Anteil durch adiabatisches Regeln von $T_{\mathrm{t}}$ gemäss $T_{\mathrm{t}}=T_{\mathrm{k}}$ zu unterdrücken oder zu einer kleinen Korrektur zu machen. Ausserdem kann die Leistung $L=0$ gewählt werden, so dass nach Gl. (2) die Temperaturänderungen nur noch durch den Wärmeaustausch bedingt sind. Bei einem isothermen Thermostaten führt das zu dem durch Gl. (2)-(5) gegebenen Temperaturausgleich. Bei variablem $T_{t}$ besteht ausserdem die Möglichkeit einer Wärmezufuhr, die durch $K\left(T_{\mathrm{k}}-T_{\mathrm{t}}\right) \mathrm{d} z$ gegeben ist. Auf diesem Prinzip beruhen die bekannten Abkühlungs- und Erwärmungsverfahren zur Bestimmung von wahren spezifischen Wärmen und Umwandlungswärmen. Ein ganz anderes Verfahren stellen die Umwandlungskalorimeter dar, bei denen $T_{\mathrm{k}}=T_{\mathrm{t}}=T_{\mathrm{u}}$ (Umwandlungstemperatur). Wegen $\mathrm{d} T_{\mathrm{k}}=0$ und $T_{\mathrm{k}}-T_{\mathrm{t}}=0$ ist sowohl die isotherme als auch die adiabatische Bedingung wenigstens näherungsweise erfüllt. Die Leistung $L$ ergibt sich dann aus der Umwandlungswärme und dem Umsatz.

Im Folgenden sollen nun die prinzipiellen Möglichkeiten erörtert werden, die sich ergeben, wenn bei konstantem oder nach einem vorgegebenen 
Programm variabel geregeltem $T_{t}$ der Kalorimetertemperatur $T_{\mathrm{k}}$ verschiedene Bedingungen auferlegt werden. Vergleichsweise einfach zu realisieren sind die Verfahren der ersten Gruppe, bei denen $T_{t}$ konstant ist.

\subsection{Verfahren mit konstanter Temperatur des Thermostaten $T_{t}$}

\subsection{1. $T_{\mathrm{k}}$ ebenfalls konstant}

1.3111. $T_{\mathrm{k}}=T_{\mathrm{t}}$ (Isotherm-adiabatisch)-Diese Bedingung läßt sich in einfacher Weise mit Umwandlungskalorimetern, insbesondere dem Verdampfungskalorimeter, realisieren, bei denen $T_{\mathbf{k}}=T_{\mathrm{t}}=T_{\mathbf{u}}$ der betreffenden Umwandlungstemperatur gewählt wird. Regelung von $T_{\mathrm{u}}$ über den Druck ermöglicht Anwendungen auf organische Reaktionen ${ }^{12-20}$ und gegebenenfalls eine einfachere Apparatur ${ }^{21}$. Die Leistung $L$ kann konstant oder variabel sein; bei Gasreaktionen über Katalysatoren hat sich jedoch Messung bei konstantem Gasstrom und konstantem Umsatz als zweckmäßig erwiesen. Der Wasserwert $W$ braucht nicht sonderlich genau bekannt zu sein, weil er wegen $\mathrm{d} \mathcal{T}_{\mathrm{k}}=0$ entweder überhaupt nicht in die Rechnung eingeht oder bei geringen Abweichungen von $\mathcal{T}_{\mathrm{k}}=$ konst nur als kleine Korrektur auftritt. Die Leistung $L$ ergibt sich nicht nach Gleichung (1), sondern aus der Umsatzgeschwindigkeit $\mathrm{d} \mathcal{N} / \mathrm{d} z$ und der Umwandlungswärme, die entsprechend genau bestimmt werden müssen.

1.3112. $T_{\mathrm{k}}-T_{\mathrm{t}}=$ Konstant (Isotherm)-Eine zweite Möglichkeit ${ }^{5,22}$ ergibt sich, wenn eine elektrisch erzeugte und entsprechend genau gemessene, am besten konstante Leistung $L$ mit einem Temperaturregler so gesteuert wird, da $\beta$ die Temperaturdifferenz $\tau_{\mathrm{k}}-T_{t}$ konstant wird. Das Verfahren läßt sich mit einer Genauigkeit von etwa 1 Prozent zweckmäßigerweise so ausführen, daß $L$ elektronisch konstant gehalten wird und die Temperaturregelung mit einem Modulationsregler erfolgt, dessen Modulationsperiode $Z_{\mathrm{m}}$ in der Größenordnung einiger ganzzahliger Sekunden liegt. Die damit synchronisierte Beobachtungsperiode $Z_{\mathrm{p}}$ wird als ganzzahliges Vielfaches von $Z_{\mathrm{m}}$ gemä $\beta Z_{\mathrm{p}}=n Z_{\mathrm{m}}$ gewählt, wobei für $n$ Werte von etwa 20 zweckmäBig sind, um Streuungen auszugleichen. Am Ende jeder Beobachtungsperiode wird die mit einer Additionsstopuhr oder einem Counter integrierte gesamte Einschaltzeit Ze der elektrischen Heizung abgelesen oder registriert, so daß der gesamte Wärmeaustausch zwischen Kalorimeter und Thermostat in der Beobachtungsperiode sich aus $Q_{\mathrm{p}}=L Z_{\mathrm{e}}$ ergibt. Reaktionswärmen $Q_{\mathrm{r}}$ folgen dann aus den Differenzen der in der Vorperiode als konstant bestimmten $Q_{\mathrm{p}}$ und den sich automatisch in der Hauptperiode anders einstellenden Einschaltezeiten $Z_{\mathrm{e}}^{\prime}$ gemä $\beta \quad Q_{\mathrm{r}}=L\left(Z_{\mathrm{e}}-Z_{\mathrm{e}}^{\prime}\right)=Q_{\mathrm{p}}-L Z_{\mathrm{e}}^{\prime}$, wodurch die kalorimetrische Messung sich in einfacher Weise auf äquidistante Registrierung der summierten Einschaltzeiten reduziert. Das Verfahren bietet den weiteren Vorzug, daß durch entsprechende Wahl von $L$ und $\left(T_{\mathrm{k}}-T_{\mathrm{t}}\right)$ eine Anpassung an die Größe der Reaktionswärme möglich ist und positive wie negative Reaktionswärmen in derselben Weise gemessen werden. Der Wasserwert tritt ebenfalls nur als Korrektur für Abweichungen von $T_{\mathrm{k}}=$ konst. in Erscheinung. Genauigkeit und Empfindlichkeit sind durch die Unsicherheit der Konstanz der Temperatur des Thermostaten gegeben. Das 


\section{KALORIMETRISCHE VERFAHREN}

Verfahren dürfte vor allem für höhere Temperaturen von Bedeutung sein, bei denen die einfachere Erwärmungsmethode wegen hoher $K$-Werte und schnellen Temperaturabfalls in der Nachperiode zu ungenau wird.

Eine weitere, spezielle Möglichkeit ${ }^{31}$ ergibt sich bei der Bestimmung von Schmelzwärmen, wobei die Abkühlung der flüssigen Probe in einem Ofen konstanter Temperatur $\mathcal{T}_{\mathrm{t}}$ bis zum Erstarrungspunkt verfolgt wird, so dass die Bedingung $T_{\mathrm{k}}=T_{\mathrm{u}}=$ konst sich aus der isothermen Umwandlung ergibt. Die Umwandlungswärme folgt dann aus der zuvor nach $\mathbf{1 . 2 2}$ bestimmten Konstanten $K$, der gemessenen Temperaturdifferenz $\mathcal{T}_{\mathbf{k}}-T_{\mathrm{t}}$ und der Dauer des Haltepunktes $Z_{\mathrm{h}}$ gemäss $Q_{\mathrm{u}}=K\left(T_{\mathrm{k}}-T_{\mathrm{t}}\right) Z_{\mathrm{h}}$.

\subsection{2. $T_{\mathrm{k}}$ variabel}

Wenn in einem Erwärmungskalorimeter eine konstante oder variable Leistung $L$ nur für eine bestimmte Dauer erzeugt wird, so resultiert eine durch die Zeitkonstanten des Kalorimeters gegebene zeitliche Änderung der Kalorimetertemperatur $T_{\mathbf{k}}$. Aus dem beobachteten Verlauf von $T_{\mathbf{k}}$ läßt sich näherungsweise der zeitliche Verlauf von $L(\mathrm{z})$ rekonstruieren ${ }^{10},{ }^{11}$. Die entwickelte Wärmemenge wird nach Gleichung (1) in zwei Anteile zerlegt, nämlich Erwärmung des Kalorimeters und Wärmeaustausch mit dem Thermostaten. Mit höheren Temperaturen und zunehmenden $K(T)$, sowie bei längerer Dauer der Wärmeentwicklung nimmt der Beitrag des Wärmeaustausches im Vergleich zum Anteil der Erwärmung schnell zu, so daß der zweite Anteil entsprechend genau bestimmt werden muß. Er kann durch adiabatisches Arbeiten unterdrückt und klein gehalten werden. Wenn der Anteil des Wärmeaustausches aber überwiegt, kann es zweckmässiger sein, umgekehrt die Erwärmung des Kalorimeters zu unterdrücken und die unter 1.1 erwähnten Methoden mit $\mathrm{d} T_{\mathrm{k}}=0 \mathrm{zu}$ verwenden.

Die Auswertung der Gleichung (1) hat bei einem Erwärmungskalorimeter die Bestimmung der beiden Apparatekonstanten $W$ und $K$ zur Voraussetzung. $W$ wird in üblicher Weise durch elektrische Eichung nach $\mathbf{1 . 2 3}$ bestimmt. Neue Kalorimeter sollten grundsätzlich auf mögliche Abhängigkeit des Wasserwertes von Dauer und Größe der Leistung $L$ geprüft werden $^{23}$. Diese Möglichkeit entfällt bei der gelegentlich angewandten Eichung durch Einwurf einer auf höhere Temperatur vorgeheizten Probe (Thermophor). Letzteres Verfahren ist aber schon darum weniger zuverlässig, weil der Wärmeinhalt der Probe nicht mit derselben Genauigkeit bekannt ist wie der einer elektrisch erzeugten Wärmemenge und weil der Wärmeverlust der Probe beim Einwurf in das Kalorimeter sich nur schwer überprüfen läßt. Die elektrische Eichung verbürgt eine weit höhere Zuverlässigkeit der Absolutwerte.

Die Bestimmung von $K$ oder $k$ erfolgt nach Abschnitt 1.21 und 1.22.

Wird der Wasserwert des Kalorimeters kleiner gehalten als der Probe, so lassen sich durch elektrische Bestimmungen des Wasserwertes des leeren und des mit der Probe beschickten Kalorimeters die spezifischen Wärmen der Probe bei verschiedenen Temperaturen unmittelbar bestimmen ${ }^{24},{ }^{25}$. Dieses Verfahren wurde bisher als nur für Temperaturen bis etwa $300^{\circ} \mathrm{C}$ brauchbar angesehen, konnte aber neuerdings auch bis $1300^{\circ} \mathrm{C}$ angewendet werden $^{26}$. 


\subsection{Verfahren mit variablem $T_{t}$}

Bei diesem Verfahren wird die Temperatur des Thermostaten nach irgendeinem Programm geregelt, wofür entweder gleichmäßige Erwärmung oder Abkühlung oder adiabatische Steuerung in Frage kommt, bei der gemäß $T_{t}=T_{k}$ der zeitliche Verlauf von $T_{t}$ durch das Programm von $T_{\mathrm{k}}$ oder umgekehrt gegeben ist. Wir erhalten in dieser Gruppe daher folgende Möglichkeiten.

\subsection{1. $T_{\mathbf{k}}=T_{\mathbf{t}}$ (Adiabatisch)}

Dies entspricht dem häufigen Fall einer adiabatischen Regelung, die sich mit heutigen Mitteln automatisch mit großer Genauigkeit erreichen läßt. Nach Gleichung (1) wird dann der Anteil des Wärmeaustausches zumindest sehr klein, so da $\beta$ die Unsicherheit des $K$-Wertes keinen sonderlichen Einffluß ausüben kann. Das adiabatische Prinzip muß aber trotzdem kritisch und mit Vorsicht angewandt werden: es ist umso unsicherer, an je weniger Stellen die Temperaturdifferenz $T_{\mathbf{k}}-T_{\mathrm{t}}$ gemessen wird. Vor allem bei plötzlich einsetzenden Reaktionen und entsprechender Erwärmung des Kalorimeters ist es keineswegs selbstverständlich, da $\beta$ der zeitliche Temperaturverlauf im Kalorimetermantel undim adiabatisch nachgesteuerten Thermostaten überall und regelmässig reproduzierbar wenigstens im Mittel der adiabatischen Bedingung genügt. Es besteht dann die Möglichkeit, da $\beta$ die Unsicherheit des $K$-Wertes zwar eliminiert, dafür aber eine größere und zudem nicht nachprüfbare Unsicherheit des zweiten Faktors $\left(T_{k}-T_{t}\right)$ eingeführt wird. Wesentlich günstiger sind dagegen die Verhältnisse bei stetigen Temperaturänderungen, wie sie bei der Bestimmung von spezifischen Wärmen durch adiabatisches Aufheizen gegeben sind. Aber auch in diesem Fall sind eine Anzahl von Vorsichtsmassnahmen zu beachten. Die relativ sicherste Methode einer Nachprüfung besteht darin, die spezifischen Wärmen bei verschiedenen Aufheizgeschwindigkeiten zu messen. Gleichung (1) zeigt nämlich, daß bei Abweichungen von der adiabatischen Bedingung die Ergebnisse von der Versuchsdauer abhängen müssen. Es empfiehlt sich ausserdem, bei einer Kontrollmessung durch ein verschiebbares Thermoelement das Kalorimeter auf uniforme Temperaturverteilung hin zu prüfen, besonders wenn es aus dünnen Blechen konstruiert wird, um einen möglichst kleinen, für die Messgenauigkeit an der Probe günstigen Wasserwert des leeren Kalorimeters zu erzielen. Nach unseren Erfahrungen können nämlich bei unzweckmässiger Anordnung der elektrischen Heizwicklungen im Kalorimeter Ueberhitzungen begrenzter Teile auftreten, die Absolutfehler von einigen Prozent bei Reproduzierbarkeit der Messung von einigen Zehntelprozent verursachen können. Der Einfluß von Abweichungen vom adiabatischen Verhalten lä $\beta t$ sich übrigens weitgehend dadurch eliminieren, daß die Messungen mit leerem und vollem Kalorimeter möglichst mit derselben Aufheizgeschwindigkeit ausgeführt werden, was allerdings im Bereich von Phasenumwandlungen nicht immer erreichbar ist.

Der Einfluß von Überhitzung ist dagegen viel schwieriger zu beseitigen, weil er mit der Größe der Leistung $L$ zunimmt, und diese bei gleicher Aufheizgeschwindigkeit im vollen Kalorimeter wesentlich größer sein muß. Es muß daher bei jedem Kalorimeter eine zweckmäßige Anordnung der Heizelemente gefunden (und kontrolliert) werden, die Überhitzung 


\section{KALORIMETRISGHE VERFAHREN}

einzelner Stellen ausschließt, besonders bei kleinem Wasserwert des Kalorimeters. Neuerdings ist es gelungen, durch adiabatische Aufheizung Messungen bei Temperaturen bis $1600^{\circ} \mathrm{C}$ auszuführen ${ }^{28}$.

Wird die Probe vom Kalorimeter aus beheizt, so bleibt ihre Temperatur hinter der seinigen zurück, d.h. die spezifische Wärme wird bei einer tieferen Temperatur bestimmt. Diese Verzögerung vermindert sich durch besseren Wärmeausgleich bei höheren Temperaturen, so daß sich eine etwas schnellere Erwärmung der Probe als im Kalorimeter ergeben wird, was bei der Berechnung der spezifischen Wärmen u.U. berücksichtigt werden muß. Zwar besteht ein Verfahren zur Berechnung der thermischen Verzögerung ${ }^{28}$, es ist aber sicherer, die Temperatur sowohl im Kalorimeter wie in der Probe zu messen.

\subsection{2. $T_{\mathrm{k}}=$ konst. $=T_{\mathrm{u}}($ Isotherm $)$}

Dieser Sonderfall tritt bei der Bestimmung von isothermen Umwandlungswärmen auf. Es gibt hier mehrere experimentelle Möglichkeiten, je nachdem, ob die Umwandlung bei sich abkühlendem oder bei aufgeheiztem Thermostat verfolgt wird.

1.3221. Bestimmung der Umwandlungswärme aus der Dauer des HaltepunktesDie Umwandlungswärme einfach proportional der Dauer des Haltepunktes zu setzen ${ }^{29}$, kann offensichtlich nicht richtig sein, weil die Temperaturdifferenz $T_{\mathrm{k}}-T_{\mathrm{t}}$ mehr oder weniger linear mit der Zeit variiert. Eine korrekte Formel wurde aus Gl. (l) abgeleitet ${ }^{30}$. Die Eichung erfolgt durch Vergleich mit bekannten Umwandlungswärmen. Dabei wird vorausgesetzt, da $K(T)$ bei Verwendung eines zweiten Tiegels und meistens bei etwas anderer Temperatur (!) denselben Wert wie bei der Eichung annehmen wird, was sicher eine grobe, nicht nachprüfbare Fehlerquelle darstellt. Zumindest müßte $K(T)$ bei verschiedenen Temperaturen bestimmt werden.

1.3222. Direkte elektrische Eichung-Bei reproduzierbarer Erwärmung des Thermostaten, beispielsweise durch konstante Regelung der Leistung eines Ofens $^{32}$, ist am Haltepunkt das Integral über $\left(T_{\mathrm{k}}-T_{\mathrm{t}}\right) \mathrm{d} z=F(0)$ ein $\mathrm{Ma} \beta$ für die Umwandlungswärme, und $Q_{\mathbf{u}}=K F(0)$. Die Bestimmung von $K$ kann dadurch umgangen ${ }^{33}$ werden, daß bei einer zweiten Messung ein Teil der Schmelzwärme mit einer Eichheizung erzeugt und genau gemessen wird; da jetzt nur ein Teil der Schmelzwärme von aussen zugeführt werden muß, ist die zweite Fläche $F(Q)$ kleiner, gemä $Q_{\mathrm{u}}-Q_{\mathrm{e}}=K F(Q)$. Daraus folgt

$$
Q_{\mathbf{u}}=Q_{\mathrm{e}} F(0) /[F(0)-F(Q)]
$$

\subsection{3. $T_{\mathrm{k}}$ variabel}

Eine wohl mehr qualitative Messung der spezifischen Wärmen nach den Methoden der D.T.A. ist möglich, wenn bei gleichmäßiger Aufheizung des Ofens $\mathcal{T}_{\mathrm{k}}$ und $\mathcal{T}_{\mathrm{t}}$ bestimmt werden. Eichung erfolgt durch Vergleich mit Proben genau bekannter (?) spezifischer Wärme, wobei dieselben Einwände wie unter 1.3221 gelten ${ }^{34-36}$. Besser wäre eine direkte Bestimmung von $K$ nach Abschnitt 1.22. 


\subsection{Apparative Ausrüstung}

Bei hohen Temperaturen müssen im wesentlichen dieselben Messungen ausgeführt werden wie bei Zimmertemperatur. So werden bei elektrischen Eichungen Strom, Spannung und Zeit bei Genauigkeiten bis 0,5 Prozent mit Zeigerinstrumenten der Güteklasse 0.2, bei höheren Ansprüchen an Genauigkeit mit Kompensationsappararen gemessen; es gibt auch halbautomatische Schaltungen ${ }^{37,38}$. Die absolute Temperatur wird entweder mit Widerstandsthermometern oder mit Thermoelementen bestimmt, deren kalte Lötstellen für genaue Messungen in einem gerührten Eisbad angebracht werden müssen ${ }^{23}$. Für die Messung der Temperaturdifferenz $T_{\mathrm{k}}-T_{\mathrm{t}}$ sind Differentialthermoelemente zweckmäßig. Die Messgenauigkeit wird bei Widerstandsthermometern bei höheren Temperaturen wohl durch die Schwierigkeit eines hinreichend konstanten Wertes des Isolationswiderstandes, und bei Thermoelementen vor allem durch die parasitären Thermokräfte begrenzt. Nach unserer Erfahrung führen Temperaturschwankungen im Labor während einer Messung zu merklich stärker streuenden Messwerten. $\mathrm{Zu}$ beachten ist ausserdem, da $\beta$ gemä $\beta$ Gl. (1) in der Regel eine Messung nur der Temperaturdifferenz $T_{k}-T_{t}$ nicht ausreicht und die absolute Temperatur $T_{\mathbf{k}}$ oder $T_{\mathrm{t}}$ gleichfalls mit entsprechender Genauigkeit bekannt sein muß. Bei Reaktionswärmen sind geringe Abweichungen von der Temperaturskala in der Regel ohne Belang, in die spezifische Wärme geht sie aber genauigkeitsbestimmend ein und muß daher im ganzen Temperaturbereich durch entsprechend präzise Eichung bestimmt werden. Mit gealterten $\mathrm{Pt}-\mathrm{Pt}, \mathrm{Rh}-\mathrm{Thermo-}$ elementen ist eine Eichung mit Kompensationsapparaten bei genau bekannten Fixpunkten auf $0.1{ }^{\circ} \mathrm{C}$ möglich, unedle Thermoelemente ${ }^{39}$ zeigen aber schon nach einmaliger Erhitzung auf $900^{\circ}$ Aenderungen von ca. $1^{\circ} \mathrm{C}$.

Bei komplizierten Apparaturen besteht die prinzipielle Schwierigkeit, da $\beta$ neben Heizspannungen der Größenordnung $10^{2} \mathrm{~V}$ Thermospannungen auf $10^{-7} \mathrm{~V}$ genau gemessen werden müssen. Die demnach erforderlichen Isolationswiderstände können aber schon ab ca. $600-700^{\circ} \mathrm{C}$ nicht mehr realisiert werden; Kriechströme lassen sich durch Erdung von Kalorimeter und Thermostat sowie durch Abschirmung der Zuleitungen und Aufstellung der Kompensatoren auf geerdete Aluminiumbleche unterbinden ${ }^{23,40}$. Ein neues Kalorimeter sollte dahingehend geprüft werden, daB Ein- oder Ausschalten irgend eines Kreises keine bleibende Aenderung oder gar ständige Schwankungen der Anzeige eines Galvanometers hervorruft; kurze induktive Stösse sind dagegen zumeist harmlos. Widerstandsthermometer werden in Verbindung mit den bekannten Brücken- oder Kompensationsschaltungen verwendet, wobei die Anforderungen an die Temperaturkonstanz der Messbrücken geprüft werden müssen. Thermokräfte werden mit thermokraftarmen Kompensatoren nach Diesselhorst gemessen, konstante Temperaturen können mit Stöpselwiderständen vorgegeben werden (einfache Kompensationsschaltung ${ }^{33}$ ). Gleichstromverstärker sind bequemer, in der Regel aber um eine Größenordnung ungenauer als einfache Galvonometer mit entsprechendem Lichtweg. Bei Temperaturen bis vielleicht $500^{\circ} \mathrm{C}$ können mehrere Kompensationswiderstände hintereinandergeschaltet werden, bei höheren muß aber jeder 


\section{KALORIMETRISCHE VERFAHREN}

Kompensationskreis getrennt aufgebaut werden, um eine gegenseitige Beeinflussung der Anzeige zu vermeiden ${ }^{23}$.

Als Öfen werden in der Regel die üblichen mit einer Heizwicklung versehenen keramischen Rohre verwendet, für höhere Temperaturen sind aber auch Molybdänrohr- ${ }^{26}$ und sogar Tammann-Öfen brauchbar ${ }^{33}$. Als Werkstoffe können Aluminium-Kupfer-Bronze oder zunderfreie Stähle verwendet werden, wobei sich aber oberhalb von $600^{\circ} \mathrm{C}$ immer ein Schutzgas empfiehlt. Bei Legierungen müssen Konzentrationen und Temperaturen gemieden werden, bei denen langsame Umwandlungen auftreten, insbesondere Ausscheidungsvorgänge, weil sie zu mangelhaft reproduzierbaren Wasserwerten führen können. Umwandlungen im Werkstoff des Kalorimeters beeinträchtigen natürlich die Genauigkeit von Messungen der spezifischen Wärme.

\subsection{Hochtemperaturthermostaten}

Die Erfahrung hat gezeigt, da $\beta$ die Genauigkeit einer kalorimetrischen Messung bei hohen Temperaturen in erster Linie von der Güte der Temperaturregelung abhängt. Elektrisch eichbare H.T.K. wurden erst durch die Entwicklung von auf einigen tausendstel Grad konstanten Thermostaten möglich. Konstanz der Temperatur läßt sich mit folgenden Mitteln erreichen.

\subsection{Konstant geregelte Spannung}

Die einfachste Methode besteht in der Verwendung einer mit Thyratrons oder Magnetverstärkern genau geregelten Spannung, mit der Ofen oder Thermostat betrieben werden. Dabei ist aber zu bedenken, daß je nach Ofencharakteristik eine Spannungskonstanz von etwa $0 \cdot 1$ Prozent bei $500^{\circ} \mathrm{C}$ eine Temperaturkonstanz von nur etwa $0.5^{\circ} \mathrm{C}$ einbringt. Eine Dämpfung nach dem Tianschen Prinzip (unter 1.3) ist daher erforderlich. Genaue Programmregelungen lassen sich so nicht verwirklichen.

\subsection{Siedepunktthermostaten}

Diese sind naturgemä $\beta$ besonders zweckmäßig bei Verdampfungskalorimetern $^{12-21}$. Die Temperatur kann zusätzlich über den Druck geregelt werden. Nachteilig ist der beschränkte Temperaturbereich: Programmregelungen über große Temperaturintervalle sind ausgeschlossen.

\subsection{Schachtelthermostaten nach Tian}

Wenn periodische Temperaturschwankungen mit einer Austauschkonstante $K$ auf einen gut temperaturleitenden Körper des Wasserwertes $W$ übergehen, werden sie im Verhältnis $W / K$ gedämpft. Durch Ineinanderschachteln von drei Ausgleichskörpern ${ }^{41,42}$ konnten so die äusseren periodischen Schwankungen von etwa 1 Prozent auf einige tausendstel reduziert werden. Derselbe Effekt wird übrigens benutzt, wenn die Temperatur des Kalorimeters mit einem Differentialthermoelement nicht gegen den Thermostaten, sondern gegen einen Ausgleichskörper gemessen wird. Solche Anordnungen sind eigentlich keine Differentialkalorimeter, sondern Tian-Thermostaten. 


\section{FRANZ EBERHARD WITTIG}

Nachteilig sind die recht großen Abmessungen solcher Thermostaten und ihre sehr träge Einstellung nach Störungen des thermischen Gleichgewichts, etwa nach Einbau einer Probe in das Kalorimeter. Zudem können sie natürlich nicht die manchmal recht erhebliche Wärmeabgabe des Kalorimeters an den Thermostaten ausregeln, so daß die Temperaturkonstanz nur vor der Messung gegeben ist und während deren Dauer Verschiebungen um einige hundertstel Grad möglich sind ${ }^{23}$. Programmregelungen sind ebenfalls ausgeschlossen. Ausserdem ist die Dämpfung umgekehrt proportional zu $K$, das sich aber erfahrungsgemä $\beta$ bei einer Temperaturerhöhung um $200^{\circ} \mathrm{G}$ etwa verdoppelt, so daß die Dämpfung eines dreiteiligen Thermostaten in diesem Intervall um den Faktor 8 vermindert werden würde; dieser Verschlechterung kann allerdings durch den Einbau von Strahlungsblechen (convection shield) begegnet werden. Systematische Abweichungen vom Sollwert, die bei Fallbügelregler durchaus in der Größenordnung von $1{ }^{\circ} \mathrm{G}$ liegen, können natürlich ebenfalls nicht ausgeregelt werden und führen daher zu systematischen Abweichungen des Thermostaten vom Sollwert.

\subsection{Modulationsregler nach Wittig, Schilling und Kemeny}

Sogenannte Zweipunktregler, wie die bekannten Fallbügelregler, führen prinzipiell zu Regelschwingungen, deren Amplitude etwa proportional der Leistungsdifferenz beider Arbeitsstufen und der Totzeit sind, die beide nicht unter ein bestimmtes Maß herabgesetzt werden können. Die Regelschwingungen lassen sich aber durch die Verwendung von $P$-Reglern, wie etwa Thyratron- oder Magnetverstärkerschaltungen, vermeiden, die aber nur bei sehr kleinen Totzeiten und Zeitkonstanten angebracht sind, da sie Impulse der Grössenordnung von 0.01 sec liefern. Bei den meist sehr großen Totzeiten thermischer Anordnungen von $c a$. $10 \mathrm{sec}$ genügen aber Impulse von einigen Sekunden, die man einfacher und billiger durch die sogenannte Linearisierung des Zweipunktreglers zu einem $P$-Regler erhält, wofür der bekannte Gouy-Modulator ein einfaches Beispiel darstell ${ }^{\mathbf{4 3}}$. Bei diesen Modulationsreglern wird durch einfache periodische Relativbewegung der Sollwertmarke und der Anzeige des Ist-Wertes ganz ähnlich wie bei der Steuerung eines Thyratrons das Verhältnis der Ein- und Ausschaltezeiten der elektrischen Leistung proportional $\mathrm{zu}$ den momentanen Abweichungen vom Sollwert verstellt. Diese Modulation läßt sich mit einfachen Mitteln durch Oszillation einer Photozelle oder durch periodische Abblendung des Lichtzeigers erzielen. Es wurde ausserdem eine rein elektronische Schaltung entwickelt, bei der Steuerimpulse erzeugt werden, deren zeitlicher Abstand über zwei durch Photozellen gesteuerte Kippkreise je nach Stand des Lichtzeigers geregelt wird, wodurch eine Linerarisierung ohne Modulation ermöglicht wird ${ }^{44}$.

Von anderer Seite ${ }^{45}$ wurde eine Einschleusung einer periodisch oszillierenden Spannung in den Thermoelement-Galvanometer-Kreis vorgeschlagen, was für Öfen durchaus brauchbar sein mag. Bei sehr genauen Temperaturregelungen bestehen aber offensichtlich die Gefahren parasitärer Thermokräfte und von Komplikationen durch Anregung des Galvanometers zu periodischen Schwingungen. 


\subsection{Der Doppelthermostat}

Mit den unter 1.54 beschriebenen Reglern kann jeder beliebige elektrische Ofen mit einfachen Galvanometern (z.B. Typ RSG 8 von Ruhstrat, Göttingen) mit einem Lichtzeiger von nur $50 \mathrm{~cm}$ auf etwa $0 \cdot 1^{\circ} \mathrm{C}$ konstant gehalten werden, so da $\beta$ durch den Tianschen Effekt die Temperaturschwankungen im Thermostatenblock nur noch etwa $0 \cdot \mathrm{pl}^{\circ} \mathrm{G}$ betragen. Für genaue Messungen muß aber die Temperatur noch um etwa eine weitere Größenordnung konstant sein. Das wird durch eine zweite Feinregelung des Thermostaten erreicht ${ }^{23}$, der auf eine um soviel höhere Temperatur eingestellt wird, da $\beta$ seine Wärmeabgabe an den Ofen um eine Größenordnung höher ist als der Maximalaustausch von Kalorimeter und Thermostat. In dieser Weise kann nämlich auch die Rückwirkung des Kalorimeters auf den Thermostaten ausgeregelt werden, was nach allen anderen Verfahren nicht möglich ist. Ein weiterer Vorteil ist natürlich dadurch gegeben, da $ß$ die Feinregelung innerhalb einer Umgebung ziemlich konstanter Temperatur $\left( \pm 0,1^{\circ} \mathrm{C}\right)$ arbeitet und die Regelstrecke im Vergleich zum äusseren Ofen eine wesentlich geringere Leistung $L$ und kleinere Zeitkonstante aufweist; auch die Totzeit dürfte etwas geringer sein. Das Verfahren des Doppelthermostaten wurde inzwischen auch für Messungen von spezifischen Wärmen übernommen ${ }^{46}$. Das axiale Temperaturgefälle scheint die Messgenauigkeit nicht so stark zu beeinflussen, wahrscheinlich weil der größte Anteil des Wärmestromes radial abfließt. Aus diesem Grunde empfiehlt es sich auch ${ }^{23}$, das Reaktionsgefä $\beta$ auf einen Asbestring zu stellen, um einen im Vergleich zum Kalorimetermantel zu grossen und zudem schlecht reproduzierbaren Wärmeaustausch mit dem Boden des Kalorimeters zu unterbinden. Trotzdem sollten in Boden und Deckel des Thermostaten Heizwicklungen angebracht werden, die vom Thermostaten-Regler über zusätzliche Kontakte geschaltet und so eingestellt werden, daß das axiale Temperaturgefälle nicht höher als $0.01^{\circ} \mathrm{C}$ ist, was am besten durch ein verschiebbares Thermoelement nachgeprüft wird. Bei höherem Aufwand könnten natürlich auch die Zusatzheizungen über besondere Regler auf konstante Temperatur von Boden und Deckel geregelt werden. Ob dieser höhere Aufwand zu einem entsprechenden Gewinn an Meßgenauigkeit führt, wurde jedoch noch nicht untersucht, weil bereits eine ausreichende Meßgenauigkeit erzielt worden war $^{23}$.

\subsection{Adiabatische Regelungen}

Die unter 1.54 und 1.55 zusammengefaßten Methoden sind nach eigenen Versuchen auch für adiabatische Reglungen bei Messungen von spezifischen Wärmen geeignet. Dazu wird das Kalorimeter mit einer elektronisch konstant gehaltenen Leistung $L$ aufgeheizt, die Temperaturdifferenz $\left(T_{\mathbf{k}}-T_{t}\right)$ durch Einstellung eines Kompensationsapparates entsprechend der in Vorversuchen ermittelten adiabatischen Bedingung vorgegeben, und die Abweichungen von diesem Sollwert dem Modulationsregler als Signal zugeführt. Ausserdem wird die Temperaturdifferenz zwischen Thermostat und Ofen auf einen zweiten Regler gegeben, der eine durch Vorversuche bestimmte günstige Temperaturdifferenz einstellt. 
Mit höheren Temperaturen nimmt aber der Leistungsbedarf von Ofen und Thermostat schnell zu, so daß die steilen Modulationsregler sehr bald ausserhalb ihres Proportionalitätsbereichs geraten würden. Es sind daher für beide Regelstrecken Nachfahrvorrichtungen für die Regeltransformatoren erforderlich. Schon lange ist das Verfahren bekannt, den Stellmotor eines Regeltransformators ebenfalls über die Kontakte des Zweipunktreglers so zu steuern, da $\beta$ bei Einschalten der höheren Leistung der Stellmotor den Regeltransformator nach höheren Spannungen verstellt, und umgekehrt. Dadurch wird offensichtlich erreicht, da $\beta$ bei zu geringer Leistung der Thermostatenheizung über die dann zunehmenden Einschaltzeiten die Leistung durch Höherregelung der Spannung vermehrt wird. Diese Methode hat allerdings den Nachteil einer überflüssig grossen Beanspruchung des Stellgliedes. Es läßt sich ausserdem nicht immer mit einfachen Mitteln erreichen, da $B$ der Stellmotor in beiden Richtungen dieselbe Drehzahl aufweist, was implizit vorausgesetzt wird.

Es ist daher besser ${ }^{44}$, mit zwei summierenden Kippkreisen die Aus- und Einschaltzeiten zu vergleichen und erst dann den Stellmotor in einer der beiden Richtungen laufen zu lassen, wenn das Verhältnis beider Zeiten einen bestimmten Erfahrungswert überschritten hat.

Bei Anwendung der unter 1.54 bis 1.56 beschriebenen Verfahren darf natürlich niemals übersehen werden, daß primär die E.M.K. eines Thermoelements auf einen konstanten Wert geregelt wird, und eine entsprechend genaue Regelung der Temperatur nur dann zustande kommt, wenn die E.M.K. für die Temperatur auch mit dieser Genauigkeit repräsentativ ist. Erfahrungsgemä $B$ wird aber die Messgenauigkeit von Reaktionskalorimetern durch langsame und stetige Verschiebungen der Temperatur, etwa durch Alterung der Thermoelemente, nicht nennenswert beeinflußt. Sogar eine allmähliche Änderung des Kompensationsstromes, die einer Temperaturänderung von einigen hundertstel Grad in 24 Stunden entsprechen würde, ist harmlos; es ist sogar besser, den Kompensationsstrom während einer Messung nicht nachzustellen. Gegebenenfalls lassen sich Änderungen der absoluten Temperatur als Korrektur in Gl. (1) einführen.

\section{SPEZIELLER TEIL}

\subsection{Messungen von Bildungswärmen}

Bestimmungen von Mischungswärmen flüssiger Metalle werden durch einfache Vereinigung beider Partner im Reaktionsgefä $\beta$ ausgeführt ${ }^{23,42,47-54}$. Bildungswärmen fester Legierungen können ebenfalls durch direkte Synthese bestimmt werden, wenn die Komponenten pulverförmig vermischt und in das Kalorimeter eingeworfen werden ${ }^{55-58}$. Dadurch wird allerdings eine gewisse Unsicherheit im Wärmeinhalt der Probe und durch den schwer erfassbaren Wärmeaustausch beim Einwurf eingeführt. Das Verfahren beschränkt sich ausserdem auf Reaktionen, die bei der fraglichen Temperatur schnell und vollständig verlaufen. Allgemeiner anwendbar ist die Methode der Lösungskalorimetrie in flüssigen Metallen ${ }^{59-71}$. Dabei treten die gleichen Schwierigkeiten auf wie bei Zimmertemperatur: es muß ein flüssiges Metall gefunden werden, in dem beide Randkompo- 
nenten und alle intermediären Phasen sich schnell und vollständig lösen, und die Bildungswärme wird als Differenz der Lösungswärmen der betreffenden Phase und der unverbundenen Randkomponenten gefunden. Aluminiumlegierungen lassen sich bis herab zu etwa $300^{\circ} \mathrm{C}$ in Zink- oder Zink-Zinnmischungen lösen ${ }^{69-71}$. In dieser Weise können noch sehr geringe Bildungswärmen von $c a .1 \mathrm{~kJ} / \mathrm{g}$-At und ihre Aenderungen mit der Konzentration von einigen $100 \mathrm{~J} / \mathrm{g}$-At in befriedigender Weise bestimmt werden.

Von Interesse ist ein weiteres Verfahren der direkten Synthese durch adiabatisches Aufheizen der unverbundenen Randkomponenten bis zum vollständigen Ablauf der Reaktion, wobei sich die Bildungswärme als Differenz gegen die besonders bestimmten spezifischen Wärmen ergibt. Diese Methode wurde mit einer einfachen Apparatur bei einer Genauigkeit von etwa 10 Prozent angewendet ${ }^{72}$; mit präzisen Apparaturen läßt sich die Messung wahrscheinlich auf eine Genauigkeit von 1 Prozent verbessern. Erfahrungen über Messung von Reaktionswärmen an anorganischen und organischen Substanzen bestehen bereits ${ }^{5},{ }^{12-21}$, und über kalorimetrische Messungen der Verformungsenergie von Metallen, u.a. durch Lösungskalorimetrie in flüssigem Zinn, liegt ein zusammenfassender Bericht vor $^{73}$.

\subsection{Messungen von spezifischen Wärmen und Umwandlungswär- men}

Bei diesen Verfahren gibt es zwei verschiedene Möglichkeiten, das Kalorimeter zu gestalten. Bei Festkörpern mit geringem Dampfdruck läßt sich nämlich schon allein die Probe durch Anbringung einer Heizwendel und der notwendigen Thermoelemente $\mathrm{zu}$ einem Kalorimeter ausbilden ${ }^{74}$. Vorteilhaft ist dabei der einfache Aufbau und der direkte Wärmeübergang von der Heizwendel auf die Probe. Von Nachteil ist dagegen die (u.U. aber nur geringe) Unsicherheit der spezifischen Wärme der keramischen Teile und Drähte, die nicht durch einen Leerversuch bestimmt werden kann. In allen anderen Fällen arbeitet man mit einem besonderen Kalorimeter, das die Heizwicklungen und Thermoelemente enthält und dessen Wasserwert $W(T)$ durch eine Meßreihe mit dem leeren Kalorimeter genau bestimmt werden kann. Ein weiterer Vorteil besteht in der Möglichkeit, die Proben schnell wechseln zu können. Nachteilig kann sich die thermische Verzögerung und mögliche Überhitzung des Kalorimeters bei hohen Leistungen auswirken. Differentialkalorimeter ${ }^{75}$ haben den zusätzlichen Nachteil, daß die $K(T)$-Werte von der Beschaffenheit der Oberfläche abhängen und ihr Unterschied nicht ohen weiteres bestimmt werden kann. Bei Relativmessungen wurde der Versuch gemacht ${ }^{76}$, diese Schwierigkeit durch bessere Wärmeleitung unter Verwendung von Wasserstoff als Füllgas zu umgehen. Bei Substanzen schlechter Wärme- und Temperaturleitfähigkeit können die thermischen Verzögerungen Schwierigkeiten bereiten. Durch Füllung solcher Substanzen in viele Bohrungen eines Aluminiumblocks liess sich jedoch bis $500^{\circ} \mathrm{C}$ eine Genauigkeit von 0.1 Prozent erzielen ${ }^{77}$; bemerkenswerterweise wird die Aufheizung öfters unterbrochen, um aus dem Gang der Vorund Nachperiode die Abweichungen vom adiabatischen Verhalten zu ermitteln. 


\subsection{Spezielle Verfahren}

Es bleiben noch die Methoden zu erwähnen, bei denen bei intermetallischen Phasen durch Abschrecken von höheren Temperaturen ein metastabiles Gleichgewicht eingefroren und anschließend durch kontinuierliches oder plötzliches Aufheizen auf eine bestimmte Temperatur nicht nur die Wärmetönung, sondern auch die Kinetik des Über ganges in das Gleichgewicht verfolgt wird. Bei kontinuierlichem Aufheizen ergeben sich die Umwandlungswärmen wieder als Differenzen gegen die spezifischen Wärmen ${ }^{78-79}$, während bei plötzlicher Erwärmung auf eine konstante Versuchstemperatur die kalorischen Effekte nach der Wärmeströmungsmethode bestimmt werden ${ }^{80-89}$.

Ein sehr summarisches Verfahren zur Bestimmung von spezifischen Wärmen ist die Drahtmethode, bei der die Probe mit kurzen Impulsen auf hohe Temperatur aufgeheizt wird und sich anschließend wieder rasch auf Zimmertemperatur abkühlt. Diese älteren ${ }^{90}$ und neueren Methoden ${ }^{91}$ zählen eigentlich nicht mehr zur Kalorimetrie bei höheren Temperaturen, weil nur die Probe auf hohe Temperaturen gebracht wird.

\section{ENTWIGKLUNG UND HEUTIGER STANDARD DER MESS- METHODIK}

An der Perfektion der Kalorimetrie bei tiefen Temperaturen und bei Zimmertemperatur sollte nicht übersehen werden, daß diese Verfahren in längeren Zeiträumen auf Grund der Erfahrungen aus sehr vielen Arbeiten entwickelt wurden. Die Zahl der Untersuchungen mit H.T.K. ist dagegen vergleichsweise noch gering. Hinreichende Kenntnisse über den zweckmäßigen Aufbau zuverlässiger Reaktionskalorimeter wurden erst in den letzten Jahren erarbeitet, so daß die Summe der vorliegenden Erfahrungen sich zwar, vor allem in letzter Zeit, erfreulich vermehrt hat, aber noch keineswegs als ausreichend angesehen werden sollte. Die Entwicklung der H.T.K. nach noch höheren Temperaturen, nach besserer absoluter und relativer Genauigkeit und nach möglichst einfachen, zweckmässigen, betriebssicheren und leistungsfähigen Anordnungen ist daher noch immer Gegenstand der Forschung, umsomehr als die Arbeiten der letzten Jahre eindeutig gezeigt haben, da 3 durch entsprechende Fortschritte Forschungsbereiche zugänglich gemacht werden, die nach älteren Verfahren nicht in befriedigender Weise untersucht werden konnten, wie beispielsweise die systematische Erforschung der Bildungswärmen in metallischen Systemen.

Die Entwicklung der H.T.K. ist eine Frage der Beherrschung der Reproduzierbarkeit der Temperaturänderungen in Thermostat und Kalorimeter und ihrer genauen Messung. Fortschritte lassen sich an der Zuverlässigkeit der elektrischen Eichung beurteilen, die daher ein wesentliches Kriterium der Güte eines Kalorimeters darstellt. Sie wurde schon vor etwa 20 Jahren bei Messungen der spezifischen Wärmen ${ }^{28,} 75$ und der Schmelzwärmen ${ }^{31-33}$ eingeführt, konnte aber erst in letzter Zeit bei H.T.K. für Reaktionswärmen angewendet werden ${ }^{23}, 42$.

Der heutige Standard der Messungen von Reaktionswärmen ist durch folgende Merkmale gegeben. 


\section{KALORIMETRISCHE VERFAHREN}

(a) Nach dem Verfahren der Erwärmungskalorimetrie können bei Temperaturen bis $1000^{\circ} \mathrm{G}$ Wasserwerte durch elektrische Eichung mit einem mittleren Fehler der Einzelmessung von 0.5-2 Prozent, je nach Temperaturbereich und Größe der Wärmemenge, bestimmt werden, invariant gegenüber Variationen der Versuchsdauer und der Wärmemenge.

(b) Ergebnisse verschiedener Autoren mit elektrisch geeichten Kalorimetern stimmen in der Regel innerhalb von 1-2 Prozent überein; etwaige größere Diskrepanzen mögen eher durch chemische als durch apparative Fehlerquellen bedingt sein.

(c) Die Apparaturen können von in 1 bis 2 Wochen angelernten Beobachtern leicht und sicher bedient werden; Fehlmessungen durch Bedienungsfehler treten praktisch nicht auf. Automatische Regelungen gestatten dem Beobachter die Konzentration auf die eigentliche Messung; je nach Arbeitszeit können 2 bis 4 Messungen täglich ausgeführt werden. Reparaturen sind selten und werden in der Regel frühestens nach einem Jahr ununterbrochener Betriebszeit fällig; die Apparaturen können verhältnismäßig leicht ausgebaut werden, so daß auch größere Reparaturen an Heizwicklungen und Thermoelementen in wenigen Tagen ausgeführt werden können. Alle Stromzuführungen werden von unten in die Apparatur eingeleitet, so da $B$ die Manipulation von Proben ungestört von oben erfolgt, und Thermoelemente können zwecks Reparatur oder Nacheichung aus der laufenden Apparatur entnommen werden.

(d) Kriechströme treten bei Temperaturen bis $1000^{\circ} \mathrm{C}$ nicht auf. Ausoder Einschalten eines beliebigen Stromkreises beeinflußt andere Messkreise schlimmstenfalls durch einen kurzen induktiven Stromstoß.

Bei der Messung von wahren spezifischen Wärmen und Umwandlungswärmen wurde bis heute folgender Stand erreicht:

(a) Ausdehnung des Temperaturbereichs bei adiabatischer Aufheizung bis $1600^{\circ} \mathrm{C}$, bei der Erwärmungsmethode bis $1300^{\circ} \mathrm{C}$;

(b) eine absolute Genauigkeit von $0 \cdot 1$ Prozent bei adiabatischer Aufheizung, bis $500^{\circ} \mathrm{C}$ auch bei Substanzen schlechter Wärmeleitfähigkeit;

(c) Entlastung des Beobachters durch partielle oder vollständige automatische Reglung des Kalorimeters einschließlich des Meßvorganges.

Im übrigen gelten die bei den Reaktionskalorimetern aufgezählten Merkmale. Die Versuchsdauer kann natürlich sehr lange sein, wenn ein größerer Temperaturbereich durch adiabatisches Aufheizen durchlaufen werden muß, weil geringe Aufheizungsgeschwindigkeiten von $c a .1^{\circ} \mathrm{C} / \mathrm{min}$ erforderlich sind. Dieser Umstand fördert die Entwicklung vollautomatischer Kalorimeter. Die Frage der Zuverlässigkeit der adiabatischen Regelung und der Sicherheit der nach dieser Methode gewonnenen Absolutwerte bedarf einer aufmerksamen, kritischen Prüfung, wenn absolute Genauigkeiten von besser als 1-2 Prozent angestrebt werden.

Diese Ausführungen mögen die experimentellen Möglichkeiten der Hochtemperaturkalorimetrie, ihre prinzipiellen Schwierigkeiten und deren weitgehende Überwindung durch neue Konstruktionen und Regelverfahren gezeigt haben. Aus den Arbeiten und Erfahrungen vieler Forscher hat 
sich so in den letzten 25 Jahren eine brauchbare experimentelle Methodik der kalorimetrischen Messungen bei höheren Temperaturen herausgebildet, die zu interessanten Anwendungen geführt hat, wie sich an der erfreulichen Zunahme von Arbeiten aus diesem Gebiet in den letzten Jahren erkennen läßt. Es sind daher noch weitere wesentliche Fortschritte zu erwarten.

\section{Literatur}

1 A. Eucken. " "Energie und Wärmeinhalt", Handbuch Experimentelle Physik, VIII/1, Akad. Verlagsges., Leipzig (1929)

${ }^{2}$ W. A. Roth, F. Becker. Kalorimetrische Methoden zur Bestimmung chemischer Reaktionswärmen, F. Vieweg \& Sohn, Braunschweig (1956)

${ }^{3}$ W. Eitel. Thermochemical Methods in Silicate Investigation, Rutgers University Press, New Brunswick (1952)

4 F. E. Wittig. "Calorimetric methods for the study of metallic systems", in The Physical Chemistry of Metallic Solutions and Intermetallic Compounds, National Physical Laboratory Symposium $\mathcal{N}$ r. 9, H.M.S.O. London (1959)

${ }^{5}$ F. E. Wittig und W. Schmatz, Z. Elektrochem., 63, 470 (1959)

${ }^{6}$ F. E. Wittig. "Kalorimetrische Methoden bei höheren Temperaturen", in Fortschr. physik. Chemie, in Vorbereitung

7 W. J. Smothers und Y. Chiang. Differential Thermal Analysis, Chemical Publ. Co., Inc., New York (1958)

${ }^{8}$ F. Weibke und O. Kubaschewski. Thermochemie der Legierungen, Springer, Berlin (1943)

${ }^{\ominus}$ O. Kubaschewski und E. Ll. Evans. Metallurgical Thermochemistry, 3rd edn., Pergamon Press, London (1958)

${ }^{10}$ W. Schmatz. Diplomarbeit, Universität München (1957)

11 E. Calvet und F. Camia. 7. chim. phys., 55, 818 (1958)

${ }^{12}$ J. R. Lacher, J. J. McKinley, G. M. Snow, L. Michel, G. Nelson und J. D. Park. $\boldsymbol{J}$. Am. Chem. Soc., 71, 1330 (1949)

${ }^{13}$ J. R. Lacher, J. J. McKinley, G. Walden, K. Lea und J. D. Park. 7. Am. Chem. Soc., 71, 1334 (1949)

${ }^{14}$ J. R. Lacher, C. H. Walden, K. R. Lea und J. D. Park. J. Am. Chem. Soc., 72, 331 (1950)

15 J. R. Lacher, T. J. Billings, D. E. Campion, K. R. Lea und J. D. Park. 7. Am. Chem. Soc., 74, 5291 (1952)

${ }^{16}$ J. R. Lacher, A. Kianpour, F. Oetting und J. D. Park. Trans. Faraday Soc., 52, 1500 (1956)

17 J. R. Lacher, E. Emery, E. Bohmfalk und J. D. Park. 7. Phys. Chem., 60, 492 (1956)

18 J. R. Lacher, L. Casali und J. D. Park. F. Phys. Chem., 60, 608 (1956)

${ }^{19}$ J. R. Lacher, A. Kianpour und J. D. Park. F. Phys. Chem., 61, 1124 (1957)

${ }^{20}$ J. R. Lacher, A. Kianpour, P. Montgommery, A. Knedler und J. D. Park. 7. Phys. Chem., 61, 1125 (1957)

${ }^{21}$ K. L. J. Tong und W. O. Kenyon. J. Am. Chem. Soc., 67, 1278 (1945); 68, 1355 (1946); 69, $1402(1947) ; 71,1925$ (1957)

${ }^{22}$ F. W. Wittig und W. Schilling. Z. Elektrochem., 65, 70 (1961)

${ }^{23}$ F. E. Wittig und F. Huber. Z. Elektrochem., 60, 1181 (1956)

${ }^{24}$ L. G. Carpenter und T. F. Harle. Proc. Roy. Soc. (London) A, 136, 243 (1932)

${ }^{25}$ L. G. Carpenter und C. J. Steward. Phil. Mag., (7), 27, 551 (1939)

${ }^{26}$ F. Krauss. Z. Metallk., 49, 386 (1958)

${ }^{27}$ I. Backhurst. 7. Iron Steel Inst. (London), 189, 124 (1958)

${ }^{28}$ H. Moser. Physik. Z., 37, 737 (1938)

${ }^{29}$ G. Tammann. Z. anorg. Chem., 43, 215 (1905); 47, 289 (1905)

${ }^{30}$ W. Plato. Z. physik. Chem., 55, 721 (1906)

${ }^{31}$ J. H. Awberry. Phil. Mag. (VII), 26, 776 (1938)

32 F. E. Wittig. Z. Metallk., 43, 157, 161 (1952)

33 F. E. Wittig. Z. Elektrochem., 54, 288 (1950)

${ }^{34}$ W. M. Cohn. J. Am. Ceram. Soc., 7, 359 (1924); 9, 206 (1926)

35 G. St. Smith, in Temperature, its Measurement and Control, Reinhold, New York (1941)

${ }^{36}$ E. S. Greiner. 7. Metals, 4, 1044 (1952)

37 F. E. Wittig. Z. Metallk., 44, 427 (1953)

${ }^{38}$ F. E. Wittig und G. Böhm. Z. Metallk., 47, 699 (1957) 


\section{KALORIMETRISGHE VERFAHREN}

${ }^{39}$ W. Hume-Rothery, J. W. Christains und W. B. Pearson. Metallurgical Equilibrium Diagrams, The Institute of Physics und Chapman \& Hall, London (1953)

40 W. P. White in Temperature, its Measurement and Control, Reinhold, New York (1941)

${ }^{41}$ A. Kelen, 7. appl. sci. res. Hague, B, 4, 309 (1954)

42 O. J. Kleppa. 7. Phys. Chem., 59, 354 (1955)

${ }^{43}$ Vgl. T. S. Sligh. 7. Am. Chem. Soc., 42, 60 (1920)

${ }^{44}$ F. E. Wittig und G. Kemeny. Chemie-Ing.-Tech., 32, 685 (1960)

${ }^{45}$ W. T. Reid in Temperature, its Measurements and Control, Reinhold, New York (1941)

${ }^{46}$ W. Jost, H. J. Oel und G. Schniedermann. Z. physik Chem. (N.F.), 17, 175 (1958)

47 O. J. Kleppa. 7. Phys. Chem., 59, 354 (1955)

${ }^{48}$ O. J. Kleppa. Acta Met., 6, 225, 233 (1958)

${ }^{49}$ R. A. Oriani und W. K. Murphy. F. Phys. Chem., 62, 199 (1958)

${ }^{50}$ F. E. Wittig und F. Huber. Z. physik. Chem. (N.F.), 18, 330 (1958)

${ }^{51}$ F. E. Wittig, E. Müller und W. Schilling. Z. Elektrochem., 62, 529 (1958)

${ }^{52}$ F. E. Wittig. Z. Elektrochem., 63, 327 (1959)

${ }^{53}$ F. E. Wittig und E. Gehring. Naturwissenschaften, 46, 200 (1959)

${ }^{54}$ F. E. Wittig und E. Müller. Z.physik. Chem., 21, 47 (1959)

${ }_{55}$ O. Kubaschewski und A. Walter. Z. Elektrochem., 45, 630, 732 (1939)

${ }^{56}$ F. Weibke und G. Schrag. Z. Elektrochem., 47, 433 (1941)

${ }^{57}$ O. Kubaschewski und F. E. Wittig. Z. Elektrochem., 47, 433 (1941)

${ }^{58}$ O. Kubaschewski und H. Villa. Z. Elektrochem., 53, 32 (1949)

${ }^{58}$ O. J. Kleppa. 7. Am. Chem. Soc., 77, 897 (1955)

${ }^{60}$ O. J. Kleppa. Acta Met., 3, 255 (1955)

${ }^{61}$ O. J. Kleppa. F. Phys. Chem., 60, 446, 842, 846, 852, 858 (1956)

${ }^{62}$ R. A. Oriani und W. K. Murphy. 7. Phys. Chem., 62, 327 (1958)

${ }^{63}$ R. L. Orr, A. Goldberg und R. Hultgren. Rev. Sci. Instr., 28, 767 (1957)

${ }^{64}$ J. L. White, R. L. Orr und R. Hultgren. Acta Met., 57, 47 (1957)

${ }_{65}$ P. D. Anderson. 7. Am. Chem. Soc., 80, 3171 (1958)

${ }^{66}$ R. L. Orr, A. Goldberg und R. Hultgren. F. Phys. Chem., 62, 325 (1958)

${ }^{67} \mathrm{~J}$. Luciat-Labry, R. L. Orr und R. Hultgren. Techn. Rep. Minerals Res. Inst., 76, Nr. 7 (1958)

${ }^{88}$ W. B. Kendall und R. Hultgren, Techn. Rep. Minerals Res. Inst., 76, Nr. 6 (1958)

69 "The heats of formation in the zeta-silver-aluminium and the epsilon-silver-zinc phases" in The Physical Chemistry of Metallic Solutions and Intermetallic Compounds, National Physical Laboratory, Symposium Nr. 9, H.M.S.O., London (1959)

${ }^{70}$ F. E. Wittig und F. Huber. Z. Elektrochem., 63, 994 (1959)

${ }^{71}$ F. E. Wittig und W. Schilling. Z. Metallk., 50, 610, (1959)

${ }^{72}$ O. Kubaschewski, W. A. Dench und V. Genta. "A high-temperature calorimeter for slow reactions", in The Physical Chemistry of Metallic Solutions and Intermetallic Compounds, National Physical Laboratory, Symposium Nr. 9, H.M.S.O., London (1959)

${ }^{73}$ A. L. Titchener und M. B. Bever. “The stored energy of cold work", Progr. in Metal Phys., 7, 247 (1958)

${ }^{74}$ T. Hirone, S. Maeda, I. Tsubokawa and N. Tsuya. Sci. Repts. Research Inst. Tohoku Univ. Ser. A, 5, 513 (1953)

${ }^{75}$ C. Sykes. Proc. Roy. Soc. (London), A, 148, 422 (1936)

${ }^{76} \mathrm{H}$. Wollenberger. Z. Metallk., 49, 629 (1958)

77 E. D. West und D. C. Ginnings. F. Research Nat. Bur. Standards, 60, 309 (1958)

${ }^{78}$ G. Bockstiegel und K. Lücke. Z. Metallk., 42, 225 (1951)

79 W. Köster und A. Schell. Z. Metallk., 43, 454 (1952)

${ }^{80}$ G. Borelius. Bull. soc. chim. France, 1949, 160

81 G. Borelius, L. E. Larsson und H. Selberg. Arkiv Fysik, 2, 161 (1950)

82 G. Borelius, S. Berglund und O. Avsan. Arkiv Fysik, 2, 551 (1950)

${ }^{83}$ G. Borelius und S. Berglund. Arkiv Fysik, 4, 173 (1951)

${ }^{84}$ G. Borelius und L. Ström. Arkiv Mat. Astr. och Fysik, A, 32, Nr. 21

${ }^{85}$ G. Borelius und K. M. Safsten. Arkiv Mat., Astr. och Fysik, B, 36, Nr. 5.

${ }^{86} \mathrm{~J}$. Nyström. Arkiv Fysik, 1, 359 (1949); 2, 151 (1950)

${ }^{87} \mathrm{~J}$. Nyström. Acta Met., 3, 182 (1955)

${ }^{88}$ O. Krisement und F. Wever. Arch. Eisenhüttenw., 25, 489 (1954); 28, 523 (1957); 29, 723 (1958) 


\section{FRANZ EBERHARD WITTIG}

s9 O. Kubaschewski und W. A. Dench. "Calorimetry", in J. O'M. Bockris, J. L. White, J. D. Mackenzie, Physico-chemical Measurements at High Temperatures, Butterworths, London (1959)

${ }^{90}$ M. von Pirani. Verh. deut. physik. Ges, 10, 1037 (1912)

${ }^{91}$ T. E. Pochapsky. Acta Met., 1, 747 (1953) 